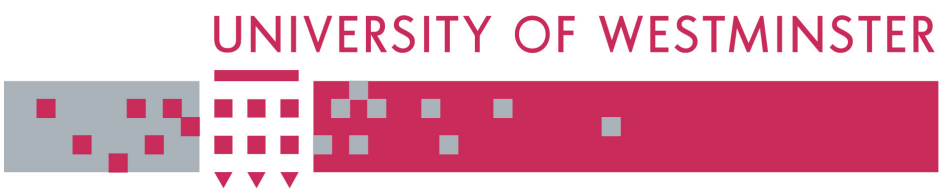

WestminsterResearch

http://www.wmin.ac.uk/westminsterresearch

\title{
A comparative study of selected classification accuracy in user profiling
}

\section{Ayse Cufoglu \\ Mahi Lohi \\ Kambiz Madani}

School of Informatics, University of Westminster

Copyright (c) [2008] IEEE. Reprinted from ICMLA '08: the Seventh International Conference on Machine Learning and Applications; San Diego, CA, USA December 11-13, 2008. IEEE, pp. 787-791. ISBN 9780769534954.

This material is posted here with permission of the IEEE. Such permission of the IEEE does not in any way imply IEEE endorsement of any of the University of Westminster's products or services. Personal use of this material is permitted. However, permission to reprint/republish this material for advertising or promotional purposes or for creating new collective works for resale or redistribution to servers or lists, or to reuse any copyrighted component of this work in other works must be obtained from the IEEE. By choosing to view this document, you agree to all provisions of the copyright laws protecting it.

The WestminsterResearch online digital archive at the University of Westminster aims to make the research output of the University available to a wider audience. Copyright and Moral Rights remain with the authors and/or copyright owners.

Users are permitted to download and/or print one copy for non-commercial private study or research. Further distribution and any use of material from within this archive for profit-making enterprises or for commercial gain is strictly forbidden.

Whilst further distribution of specific materials from within this archive is forbidden, you may freely distribute the URL of the University of Westminster Eprints (http://www.wmin.ac.uk/westminsterresearch).

In case of abuse or copyright appearing without permission e-mail wattsn@wmin.ac.uk. 


\title{
A Comparative Study of Selected Classification Accuracy in User Profiling
}

\author{
Ayse Cufoglu, Mahi Lohi and Kambiz Madani \\ Department of Electronics, Communication and Software Engineering \\ University of Westminster \\ London, UK \\ \{A.Cufoglu1, lohim, madanik\}@,westminster.ac.uk
}

\begin{abstract}
In recent years the used of personalization in service provisioning applications has been very popular. However, effective personalization cannot be achieved without accurate user profiles. A number of classification algorithms have been used to classify user related information to create accurate user profiles. In this study four different classification algorithms which are; Naïve Bayesian (NB), Bayesian Networks (BN), Lazy Learning of Bayesian Rules (LBR) and Instance-Based Learner (IB1) are compared using a set of user profile data. According to our simulation results $\mathrm{NB}$ and IB1 classifiers have the highest classification accuracy with the lowest error rate.
\end{abstract}

\section{INTRODUCTION}

In literature there are various definitions for user profile [1][3]. However, we can define it as the description of the user interests, characteristics, behaviors and preferences. User profiling is the practice of gathering, organizing and interpreting the user profile information [4]-[6].

As previously mentioned, user profiles include various information about each user. For instance, if we assume that user profiles are three dimensional matrices, each dimension of the matrix will represent a particular user related information such as; personal profile data (demographic profile data), interests profile data and preference profile data.

There are few works that compare some of the classification algorithms. In [8] Huang et al. compared AUC - known as the area under the ROC (Receiver Operating Characteristics) Curveand accuracy of Naïve Bayes, Decision Trees and Support Vector Machine (SVM). Authors claimed that AUC is a better measure of accuracy with respect to the degree of discriminancy and consistency. According to their experimental results Naïve Bayesian, Decision Trees (C4.5, C4.4) and SVM are very similar with respect to the average predictive accuracy. In addition, Naïve Bayesian, C4.4 [19] and SVM have a similar average predictive AUC which is significantly higher than C4.5.

In another work Wang et al. [9] compared and constructed the relative performance of LBR and TAN (Tree Augmented Naïve Bayesian). In this work TAN algorithm approximates interactions between attributes by using a tree structures imposed on Naïve Bayesian structure [10]. LBR is desirable when small numbers of objects to be classified while TAN is desirable when large numbers of objects to be classified [14].

In [15] authors proposed Lazy Naïve Bayesian (LNB) algorithm and compare it with SNNB (Selective Neighborhood based Naïve Bayesian), LWNB (Locally Weighted Naïve Bayesian) and Lazy of Bayesian Rules (LBR). According to the authors, SNNB and LWNB improve classification accuracy of Naïve Bayesian (NB) while LNB improve ranking accuracy of NB. LNB spends no effort during training time and delays all computation until classification time. LNB learning algorithm deals with Naïve Bayes' unrealistic attribute conditional independence assumption by cloning each training instance to produce an expanded training instance. Based on the AUC measurement SNNB and LWNB can not significantly improve the NB, and LBR performs worse than NB. According to authors' experiments, LNB is slightly better than NB and C4.4 Decision Tree, with respect to the accuracy, robustness and stability.

In another work Zhang et al. [18] compared the ranking performance of NB and DT (C4.4) classifiers. The experiments conducted with using 15 dataset from UCI data repository [16] .According to the experimental results NB algorithm outperforms the $\mathrm{C} 4.4$ algorithm in 8 datasets, ties in 3 datasets and loses in 4 dataset. The average AUC of $\mathrm{NB}$ is $90.36 \%$ which is substantially higher than the average $85.25 \%$ of C4.4. Considering these results, authors argue that NB performs well in ranking, just as it does in classification.

This study is aimed to find the best classification algorithm for user profiling process.

In this paper Naïve Bayesian networks (NB), Bayesian Networks (BN), Instance-Based Learner (IB1) and Lazy Learning of Bayesian Rules (LBR) classification algorithms are compared in terms of classification accuracy of the user profile data. These four algorithms have been chosen since BN and NB algorithms are two of the most successful algorithms in Machine Learning (ML) and Data Mining (DM) fields; IB1 has never been considered for such a research work with BN, LBR and $\mathrm{NB}$; and LBR is one of the best NB algorithms. 


\section{NB, BN, LBR AND IB1 ALGORITHMS}

The following section describes the NB, BN, LBR and IB1 classification algorithms.

Bayesian networks are probability based and are used for the reasoning and the decision making in uncertainty, and heavily rely on bayes' rule [7]. Bayes' rule can be defined as follows [7],

- Assume $A_{i}$ attributes where $\mathrm{i}=1,2,3, \ldots, \mathrm{n}$, and which take values $a_{i}$ where $\mathrm{i}=1,2,3, \ldots, \mathrm{n}$.

- Assume $\mathrm{C}$ as class label and $E=\left(a_{1}, a_{2}, \ldots, a_{n}\right)$ as unclassified test instance.

- $\mathrm{E}$ will be classified into class $\mathrm{C}$ with the maximum posterior class probability $P(C \mid E)$,

$$
P(C \mid E)=\arg \max _{C} P(C) P(E \mid C)
$$

Bayesian Networks can represent uncertain attribute dependencies, however it has been proven that learning optimal Bayesian network is NP (Non-deterministic Polynomial) hard [15].

Naïve Bayesian Classifier is one of the Bayesian Classifier techniques which also known as the state-of-the-art of the Bayesian Classifiers. In many works it has been proven that Naiive Bayesian classifiers are one of the most computationally efficient and simple algorithms for ML and DM applications [9] - [12]. Naïve Bayesian classifiers assume that all attributes within the same class are independent given the class label. Based on this assumption, the Bayesian rule has been modified as follows to define the Naïve Bayesian rule [7],

$$
P(C \mid E)=\arg \max _{C} P(C) \prod_{i=1}^{n} P\left(A_{i} \mid C\right)
$$

Naïve Bayesian classifiers are used within many interactive applications because of its efficiency and effectiveness. However, because of its naïve conditional independence assumption, optimal accuracy can not be achieved. LBR is one of the lazy learning algorithms that have been proposed to improve the accuracy performance of Naïve Bayesian classifier. LBR algorithm can be thought of as applying Lazy Learning techniques to Naïve Bayesian rule [9]. At the classification time of each test instance, LBR algorithm builds the most appropriate Bayesian rule for the test instance. Following formula shows the LBR Bayes rule that used for classification [17],

$$
P\left(C_{i} \mid V_{1} \wedge V_{2}\right)=P\left(C_{1} \mid V_{2}\right) P\left(V_{1} \mid C_{i} \wedge V_{2}\right) / P\left(V_{1} \mid V_{2}\right)
$$

Here we assume that $V_{1}$ and $V_{2}$ are any two conjunction of attribute values and $V=\left(a_{1}, a_{2}, \ldots, a_{i}\right)$ is an attribute vector. At each instance classification time each attribute values $a_{i}$ from $V$ are allocated to exactly $V_{1}$ or $V_{2}$ such that $V_{1}=\left(A_{1}, A_{2}, \ldots, A_{n}\right)$ and $V_{2}=\left(A_{n+1}, A_{n+2}, \ldots, A_{t}\right)$ where $A_{i}=a_{i}$.
IB1 or IBL (Instance-Based Learning) is one of the other classifiers and it is a comprehensive form of the Nearest Neighbor algorithm [13] [14]. IB1 generates classification predictions using only specific instances. Unlike Nearest Neighbor algorithm, IB1 normalizes its attributes' ranges, processes instances incrementally and has a simple policy for tolerating missing values [14]. IB1 uses simple normalized Euclidean distance (similarity) function to yield graded matches between training instance and given test instance [13]. Following function is the similarity that is used within IB1 algorithm [14],

$$
\operatorname{Similarity}(x, y)=-\sqrt{\sum_{i=1}^{n} f\left(x_{i}, y_{i}\right)}
$$

Here, instances are represented by $\mathrm{n}$ attributes where $f\left(x_{i}, y_{i}\right)=\left(x_{i}-y_{i}\right)^{2}$ represents numeric valued attributes and $f\left(x_{i}, y_{i}\right)=\left(x_{i} \neq y_{i}\right)$ represents Boolean and symbolic attributes.

\section{ClASSIFICATION ACCURACY}

In this section we compare the results of four classifiers (NB, $\mathrm{BN}, \mathrm{LBR}$ and IB1). The simulations conducted twice with using two different datasets. The first dataset reflects the users' personal information (demographic data) while the second dataset incorporates the user's personal information with the user's interests and preferences information. As a demographic profile data, UCI's adult dataset [16] has been modified and used. All simulations were performed in the Weka machine learning platform that provide a workbench which consist of collection of implemented popular learning schemes that can be used for practical data mining and machine learning works [13].

Below we highlighted the procedure for the simulations;

- Datasets have been converted into Weka readable ".cvs" format (see Table I). First 20 instances of the UCI's adult dataset have been chosen for the simulations.

- The first dataset, demographic user profile, includes 20 instances and 10 attributes (see Table I). These attributes are; Age, Work-class, Education, Education-num, Marital-status, Occupation, Relationship, Race, Sex and Native-country. In table I, missing values indicated with “?” symbol.

- The second dataset, extended user profile, consists of 20 instances and 18 attributes. These attributes are; Age, Workclass, Final-weight, Education, Education-num, Maritalstatus, Occupation, Relationship, Race, Sex, Native-country, capital-gain, capital-loss, Hours-per-week, Interest-music, interest-book, interest-sport and Preference-sound. 
Table I. Personal User Profile Data in “.cvs” Format

\begin{tabular}{|c|c|c|c|c|c|c|c|c|c|}
\hline Age & Work-class & Education & $\begin{array}{l}\text { Education- } \\
\text { num }\end{array}$ & Marital status & Occupation & Relationship & Race & Sex & Native country \\
\hline 25 & Private & $11^{\text {th }}$ & 7 & Never-married & Machine-op-inspct & Own-child & Black & Male & United-states \\
\hline 38 & Private & HS-grad & 9 & Married-civ-spouse & Farming-fishing & Husband & White & Male & United-states \\
\hline 28 & Local-gov & Assoc-acdm & 12 & Married-civ-spouse & Protective-serv & Husband & White & Male & United-states \\
\hline 44 & Private & Some-collage & 10 & Married-civ-spouse & Machine-op-inspct & Husband & Black & Male & United-states \\
\hline 18 & $?$ & Some-collage & 10 & Never-married & ? & Own-child & White & Female & United-states \\
\hline 34 & Private & $10^{\text {th }}$ & 6 & Never-married & Other-service & Not-in-family & White & Male & United-states \\
\hline 29 & $?$ & Hs-grad & 9 & Never-married & $?$ & Unmarried & Black & Male & United-states \\
\hline 63 & $\begin{array}{l}\text { Self-emp-not- } \\
\text { inc }\end{array}$ & Prof-school & 15 & Married-civ-spouse & Prof-specialty & Husband & White & Male & United-states \\
\hline 24 & Private & Some-collage & 10 & Never-married & Other-service & Unmarried & White & Female & United-states \\
\hline 55 & Private & $7^{\text {th }}-8^{\text {th }}$ & 4 & Married-civ-spouse & Craft-repair & Husband & White & Male & United-states \\
\hline 65 & Private & HS-grad & 9 & Married-civ-spouse & Machine-op-inspct & Husband & White & Male & United-states \\
\hline 36 & Federal-gov & Bachelors & 13 & Married-civ-spouse & Adm-clerical & Husband & White & Male & United-states \\
\hline 26 & Private & HS-grad & 9 & Never-married & Adm-clerical & Not-in-family & White & Female & United-states \\
\hline 58 & $?$ & HS-grad & 9 & Married-civ-spouse & $?$ & Husband & White & Male & United-states \\
\hline 48 & Private & HS-grad & 9 & Married-civ-spouse & Machine-op-inspct & Husband & White & Male & United-states \\
\hline 43 & Private & Masters & 14 & Married-civ-spouse & Exec-managerial & Husband & White & Male & United-states \\
\hline 20 & State-gov & Some-collage & 10 & Never-married & Other-service & Own-child & White & Male & United-states \\
\hline 43 & Private & HS-grad & 9 & Married-civ-spouse & Adm-clerical & Wife & White & Female & United-states \\
\hline 37 & Private & HS-grad & 9 & Widowed & Machine-op-inspct & Unmarried & White & Female & United-states \\
\hline 40 & Private & Doctorate & 16 & Married-civ-spouse & Prof-specialty & Husband & $\begin{array}{l}\text { Asian- } \\
\text { Pac. }\end{array}$ & Male & $?$ \\
\hline
\end{tabular}

- We chose 10 fold cross-validation as a test mode where 10 pairs of training sets and testing sets are created. All previously mentioned classification algorithms run on the same training sets and have been tested on the same testing sets to obtain the classification accuracy.

- Unlike other aforementioned three algorithms, LBR cannot handle numeric attributes. Therefore, before we do simulations with LBR, we normalized and binarised the attribute values of both datasets using unsupervised attribute filters "Normalized" and "Numeric-To-Binary".

\section{A. Comparison of the Results}

We conducted the first simulations on demographic user profile dataset to compare $\mathrm{NB}, \mathrm{BN}, \mathrm{LBR}$ and IB1 classifiers using classification accuracy as evaluation criterion. Table II demonstrates the classification accuracy results of these four classifiers. As we can see from table II, NB and IB1 classifiers have the result of $95 \%$ where 19 dataset instances have been classified correctly and 1 instance has been classified incorrectly. Moreover, with the second highest result that is $90 \%$, LBR classifier followed the outcome of NB and IB1 algorithms. Bayesian classifier result is the lowest which is $85 \%$ (17 correctly classified and 3 incorrectly classified instances). Here, both NB and IB1 outperform the LBR and BN classifiers in terms of classification accuracy.

Table III shows that precision of the four classification algorithms are very similar.
Table II. Classification Accuracy Test Results (Simulation1)

\begin{tabular}{|c|c|c|}
\hline Classifier & $\begin{array}{c}\text { Correctly } \\
\text { classified } \\
\text { instances }\end{array}$ & $\begin{array}{c}\text { Incorrectly } \\
\text { classified } \\
\text { instances }\end{array}$ \\
\hline NB & $19(95 \%)$ & $1(5 \%)$ \\
\hline IB1 & $19(95 \%)$ & $1(5 \%)$ \\
\hline LBR & $18(90 \%)$ & $2(10 \%)$ \\
\hline BN & $17(85 \%)$ & $3(15 \%)$ \\
\hline
\end{tabular}

Table III. Classifiers vs. Precision

\begin{tabular}{|c|c|}
\hline Classifier & Precision \\
\hline NB & 0.95 \\
\hline IB1 & 0.95 \\
\hline LBR & 0.947 \\
\hline BN & 0.944 \\
\hline
\end{tabular}


Fig. 1 shows the error rate results. Here four different parameters are used to represent the error rate of the four classification algorithms. These are; Mean Absolute Error (MAE), Root Mean Squared Error (RMSE), Relative Absolute Error (RAE) and Root Relative Squared Error (RRSE). It shows that NB and IB1 classifiers have the lowest error rate. Furthermore, BN classifier has the highest error rate and the difference is more in RRSE and RAE measurements, knowing that low error rate cause high accuracy or vice versa. Based on the above classification accuracy results (see Table II), the BN classifier demonstrates the highest error rate (see Fig 1).

In order to compare the classification accuracy performance of the NB, BN, LBR and IB1 classifiers with complete user profile data, a second simulation was performed on the extended user profile dataset. During the second simulation we have observed the following;

- The classification accuracy performance of the BN classifier was $80 \%$. Therefore, when this result is compared with the first simulation we can see that BN classifiers performance degreases $5 \%$ from $85 \%$ to $80 \%$. On the other hand, for NB, IB1 and LBR classifiers, first simulation results have remained the same during the second simulations (see Table IV). Therefore, NB and IB1 classification algorithms keep performing well with bigger user profile dataset.

According to our simulation results $\mathrm{NB}$ outperforms $\mathrm{BN}$ classifier. This is due to the fact that NB classifier assumes that class attributes within the same class are conditionally independent given the class label. Furthermore, we know that LBR classifier proposed to improve the performance of NB classifier by applying the lazy algorithm on the NB classifier. However, our results show that LBR classifier performs lower classification accuracy than NB.

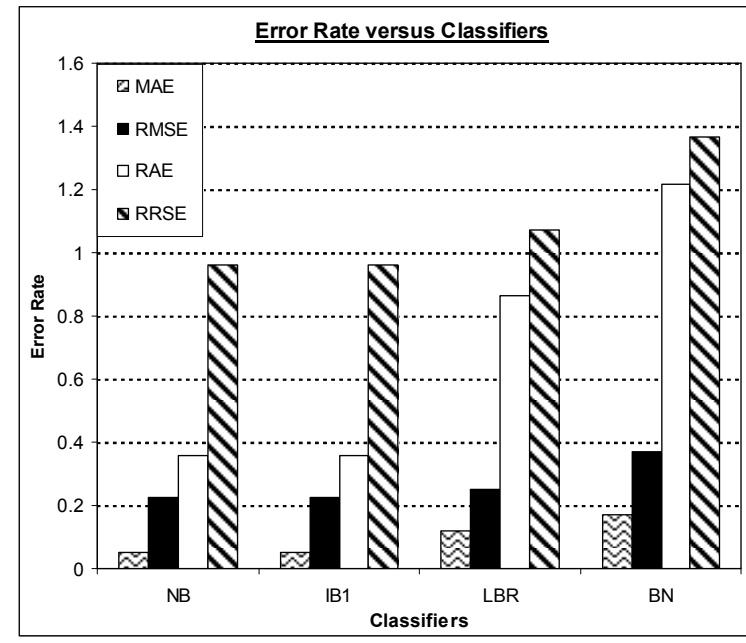

Figure1. Error rate measures of Classifiers (Simulation 1)
Table IV. Classification Accuracy Test Results (Simulation 2)

\begin{tabular}{|c|c|c|}
\hline Classifier & $\begin{array}{c}\text { Correctly } \\
\text { classified } \\
\text { instances }\end{array}$ & $\begin{array}{c}\text { Incorrectly } \\
\text { classified } \\
\text { instances }\end{array}$ \\
\hline NB & $19(95 \%)$ & $1(5 \%)$ \\
\hline IB1 & $19(95 \%)$ & $1(5 \%)$ \\
\hline LBR & $18(90 \%)$ & $2(10 \%)$ \\
\hline BN & $16(80 \%)$ & $4(20 \%)$ \\
\hline
\end{tabular}

- Fig. 2 shows the error rate results of the four classifiers respectively. According to these results, in the second simulations RAE of LBR and BN classifiers have increased significantly. This increment is much more in BN classifier where RAE increases from $121 \%$ to $162 \%$.

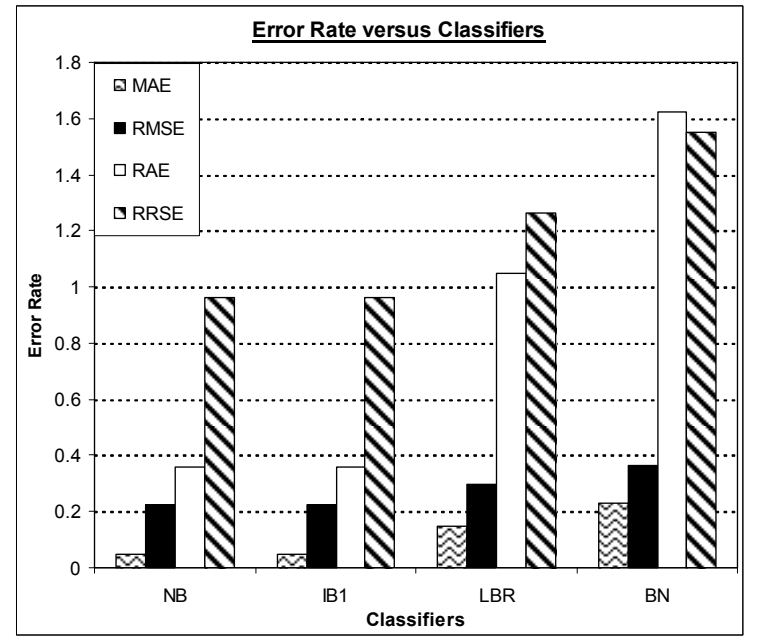

Figure2. Error rate measures of Classifiers (Simulation 2)

\section{CONCLUSION}

In this paper we evaluated classification accuracy of four classification algorithms (BN, NB LBR and IB1). All simulations were performed in Weka [13] machine learning platform. Moreover, UCI adult dataset [16] has been modified and used as a demographic user profile data. The aim of these simulations was to find the best classification algorithm that has a high classification accuracy performance on the user profile data. According to the simulation results $\mathrm{NB}$ and IB1 classifiers perform the best classification on user related information. Furthermore, LBR shows similar results to NB and IB1 that are slightly different from $\mathrm{BN}$. This indicates that NB and IB1 classification algorithms should be favored over LBR and BN classifiers in the personalization applications especially when the classification accuracy performance is important. In our future work, we will compare the well known DT and SVM classifiers with IB1 and NB classifiers with respect to classification accuracy performance on relatively larger user profile dataset. 


\section{REFERENCES}

[1] Araniti G., Meo P. D., Iera A. and Ursino D. (2003) "Adaptive controlling the QoS of multimedia wireless applications through user profiling techniques", IEEE Journal on selected areas in communication, Vol. 21, No. 10, Page(s) 1546-1556.

[2] ETSI (2005) Human Factors (HF). "User profile management" [Online] Available from: http://webapp.etsi.org

[3] Kuflik T. and Shoval P. (2000) "Generation of user profiles for information filtering- research agenda" [Online] Available from: http://delivery.acm.org

[4] Henczel S. ( 2004) "Creating user profiles to improve information quality" Factiva, Vol. 28, No 3, Page(s) 30, [Online] Available From: http://global.factiva.com/ha/default.aspx

[5] Usability by Design (2004) User Profiling [Online] Available from: www.usability.uk.com/glossary/user-profiling.htm

[6] Open Interface LTD (-) User Profiling [Online] Available from: http://www.openinterface.ie/development/design user.html

[7] Jensen F.V. (1993) Introduction to Bayesian Networks. Denmark, Hugin Expert A/S.

[8] Huang J., Lu J. and X. Ling C. (2003) "Comparing naïve bayes, decision trees, and SVM with AUC and Accuracy" . 3rd conferences on Data Mining, IEEE, ICDM 2003, Page(s) 553 - 556

[9] Wang Z. and I. Webb G. (2002) "Comparison of lazy bayesian rule and tree-augmented bayesian learning".IEEE conference on Data Mining, ICDM 2002, Page(s): 490 - 497.

[10] Shi Z., Huang Y. and Zhang S. (2005) "Fisher score based naive bayesian classifier". International conference on Neural Networks and Brain, IEEE, ICNN\&B 2005, Page(s) 1616- 1621.

[11] Xie Z. and Zhang Q. (2004) "A study of selective neigbourhood-based naïve bayes for efficient lazy learning". 16th IEEE International Conference on Tools with Artificial Intelligence, ICTAI 2004.
[12] Santafe G., Loranzo J.A. and Larranaga P. (2006) "Bayesian model averaging of naive bayes for clustering" .IEEE Transactions on Systems, Man, and Cybernetics, Vol 36, No 5, Page(s) $1149-1161$.

[13] Ian H. Witten and Frank E. (2005) Data Mining: Practical machine learning tools and techniques, 2nd Edition, Morgan Kaufmann, San Francisco, 2005.

[14] David W. Aha, Kibler D. and K. Albert M. (1991) “ Instance-based learning algorithms". Machine Learning journal, Vol 1, No 6, ISDN 15730565, Page(s):37-66. [Online] Available from: http://www.springerlink.com/content/kn127378pg361187/fulltext.pdf

[15] Jiang L. and Guo Y. (2005) "Learning lazy Naïve Bayesian classifier for ranking". 17th IEEE conference on tools with artificial intelligence, IEEE 2005, Page(s) 5pp.

[16] Asuncion A. and Newman D.J. (2007) UCI Machine Learning Repository Irvine, CA: University of California, School of Information and Computer Science. [Online] Available from: $\mathrm{http}: / /$ www.ics.uci.edu/ mlearn/MLRepository.html

[17] Zheng Z. and Webb G. I. (2000) "Lazy learning of bayesian rules" Machine Learning, Vol 41, No 1, Page(s): 53-87. [Online] Available from: http://www.csse.monash.edu.au/ webb/Files/ZhengWebb00.pdf

[18] Zhang H. and Su J. (2004) "Naive bayesian classifiers for ranking" . 15th European Conference on Machine Learning, ECML 2004. [Online] Available http://www.cs.unb.ca/profs/hzhang/publications/NBRanking.pdf

[19] Provost F. and Domingos P. (2003) "Tree induction for probability based ranking". Kluwer Academic Publishers, Vol 52 , No 3 [Online] Available from: http://portal.acm.org 